\title{
Experimental study on onset of Vortex-Induced Vibration of a pipeline near a seabed
}

\author{
Z.P. Zang \& F.P. Gao \\ Key Laboratory for Mechanics in Fluid Solid Coupling System, Institute of Mechanics, \\ Chinese Academy of Sciences, Beijing, China
}

\begin{abstract}
The critical flow velocity for onset of Vortex-Induced Vibration (VIV) of a pipeline near a rigid or a scoured seabed was experimentally investigated. The model pipe was initially positioned at a small gap to a rigid seabed or partially embedded in a silty seabed. The value of the embedment/gap-to-diameter ratio was changed from -0.2 to 1.0. A statistic form of the flow velocity, which is composed of the mean value and the Root-Mean-Square Deviation (RMSD) of the flow velocity, is applied in quantifying the onset condition of VIV. It was found that the onset of VIV is attributed to the joint contributions of both the mean flow and the turbulent component of the flow. The correlation of the critical reduced velocity with the initial position of the pipeline and the scour depth were concluded based on the experimental results.
\end{abstract}

\section{INTRODUCTION}

Free spans often occur along a pipeline as the results of either the local scour or the unevenness of the seabed. VIV of a pipeline, which has been considered as one of the most important reasons for a pipeline failure, may occur when the ocean flow crossing the pipeline is larger than a certain value. Most existing studies on VIV were mainly focused on the vibration amplitude and frequency for a wall-free (Feng, 1968; Sarpkaya, 2004; Govardhan \& Williamson, 2006; Vandiver, 2012) or near-bed cylinder (Jacobsen et al, 1984; Yang et al, 2009; Zang \& Gao, 2014). However, the critical condition for the onset of VIV is also a crucial parameter in practical engineering, as it is closely related to the allowable length for avoiding VIV of the spanning pipeline.

The proximity of the seabed may have a significant effect on the onset of VIV due to the suppression of vortex shedding from the pipe by the bottom.

The near-wall effects on vortex shedding and hydrodynamic loading of a single pipeline have been studied experimentally or numerically by many researchers. The PIV technique was employed recently by Wang \& Tan (2008) and Zang et al (2013) to measure the wake flow fields around a single circular cylinder near a plane boundary. The lee-wake patterns and the characteristics of vortex shedding from the cylinder have been examined quantitatively.

There are also a few efforts on the coupling effect of the VIV of pipeline and the soil scour. Sumer et al (1988) and Gao et al (2006) studied interactions between a vibrating pipeline and an erodible bed experimentally. In both investigations, the pipeline was allowed to vibrate in the transverse direction. It was found that the VIV does not happen until the scour depth below the pipeline is large enough; scour affects not only the amplitude of the pipeline vibration but also its frequency. Zhao \& Cheng (2010) shown that the scour pit underneath a two-degree-of-freedom vibrating pipeline is deeper than that under a pipeline vibrating only in the transverse flow direction. The water depth has weak effect on the scour depth, but the time scale of the scour. Till now, there were still few investigations on the onset of VIV, especially for a pipeline near a scoured seabed.

In this study, the transverse VIV of a pipeline close to a rigid seabed and an erodible seabed in a steady flow was physically modeled. Based on the analyses of the flow velocity, vibration amplitudes and scour profile, the relationships between the critical reduced velocity for onset of VIV, the initial position of the pipeline and the scour depth, were obtained.

\section{EXPERIMENTAL SETUP AND TEST CONDITIONS}

The experiments were conducted in a fluid-structuresoil interaction flume $(52.0 \mathrm{~m}$ long, $1.0 \mathrm{~m}$ wide and $1.5 \mathrm{~m}$ high) at the Institute of Mechanics, Chinese Academy of Sciences. A large soil box $(6.0 \mathrm{~m}$ in length, $1.0 \mathrm{~m}$ in width and $0.6 \mathrm{~m}$ in depth) is located in the middle section of the flume to simulate the seabed. A hydro-elastic facility, which was updated from the one ever used by Zang \& Gao (2014), was utilized for physical modeling the onset of VIV of a pipeline. The model pipe was attached to the supporting frame by two linear guide rails and two sets of springs. The 


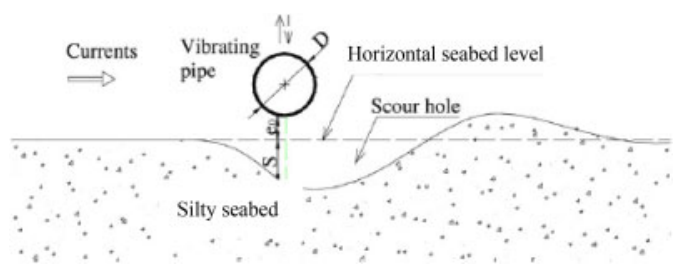

Figure 1. Sketch of VIV of a pipeline close to a seabed (adopted from Gao et al, 2006).

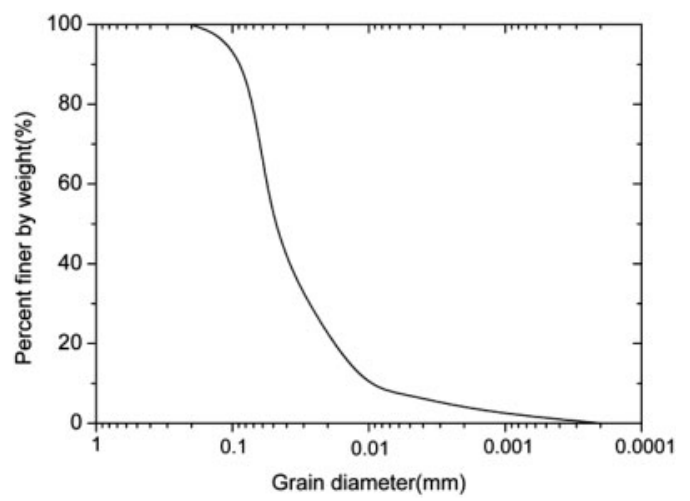

Figure 2. Particle Size Distribution of silt used in experiments.

linear guide rails can move smoothly along the two bearings, which are fixed on the frames. In the present study, only transverse vibration was simulated with the hydro-elastic facility. The model pipe is made of a plexiglass tube with the outer diameter $D=80 \mathrm{~mm}$ and the length $L=980 \mathrm{~mm}$. The water depth $(h)$ in the flume was maintained at $0.4 \mathrm{~m}$ for all tests.

The experiments are composed of two parts: VIVs of a pipeline close to a rigid seabed and those close to an erodible seabed. For the second part of the experiments, silt with the median size of $d_{50}=0.047 \mathrm{~mm}$ was used to simulate the seabed. The model pipe was initially placed above the rigid bed or embedded in the silty seabed. The sketch of VIV of a pipeline close to a rigid/silty seabed is shown in Figure $1 . e_{0}$ denotes the initial position of the pipeline, which is defined as the distance between the bottom of the pipeline and the horizontal seabed level, which ranges in $-0.2 \sim 1.0$ in this study. $S$ denotes the scour depth below the pipeline. The grain size distribution of the test silt is shown in Figure 2. The scour depth development was recorded with a video camera.

The oscillation of the pipe was measured with a Laser Displacement Transducer (LDT) mounted on the supporting frame. The natural frequency of the model pipeline $f_{n}$ was obtained by spectrum analyses of free-decay tests in the still water. The damping factor $\zeta$ was determined with the conventional free-decay testing method, i.e. applying a given excitation to the structure, then recording the vertical oscillation with the LDT. For slightly damped structures, the damping factor can be estimated with $\zeta=\ln \left(A_{i} / A_{i+n}\right) /(2 \pi n)$, where $A_{i}$ is the initial amplitude of pipe vibrations, $A_{i+n}$ is the amplitude after $n$ cycles. Note that the measured damping factor includes both structural and hydrodynamic sources. In this study, the natural frequency of the vibration system $f_{n}$ is $0.665 \mathrm{~Hz}$ and the damping ratio $\zeta$ is 0.081 .

The free stream flow velocity was measured with an Acoustic Doppler Velocimetry (ADV), which was placed at $3.0 \mathrm{~m}$ upstream the pipeline and $0.12 \mathrm{~m}$ above the flume bottom. The tests were conducted by increasing the flow velocity from 0 and gradually to the critical flow velocity, at which the pipeline starts to vibrate. For most of tests, a flow ramp-up rate $a=2.0 \times 10^{-4} \mathrm{~m} / \mathrm{s}^{-2}$, which is a representative of the typical storm in oceans, was employed to initiate the onset of VIV. There are also some tests to study the effect of the ramp-up rate on the onset of VIV, for which the ramp-up rate ranges in $1.0 \sim 7.0 \times 10^{-4} \mathrm{~m} / \mathrm{s}^{-2}$. The Reynolds number $R e$ $(=U D / v, v$ is the kinematic viscosity of the water, taken as $1.0 \times 10^{6} \mathrm{~m}^{2} / \mathrm{s}$ ) at the onset of VIV ranges in $1.5 \sim 3.5 \times 10^{4}$ in this study. All test data, including the flow velocity, the vibration amplitude and the scour profiles, were recorded synchronously.

\section{RESULTS AND DISCUSSION}

\subsection{Statistic analysis of flow velocity for onset of VIV}

The Root-Mean-Square values of the lift force were analyzed as the key parameter by Lei et al (2000) to study the flow characteristics of a pipeline and they observed that there are three different flow regimes for the near-wall pipe. Here, a statistic form of the flow velocity $U$, which is composed of the mean flow velocity $\bar{u}$ and the Root-Mean-Square Deviation (RMSD) of the flow $u^{\prime}$, was defined, namely,

$U=\bar{u}+u^{\prime}$

$u^{\prime}=\operatorname{RMSD}(u)=\sqrt{\frac{\sum_{i=1}^{n}(u-\bar{u})^{2}}{n}}$

where $u$ is the measured flow velocity, $n$ is set at 250, which stands for a time segment of $5 \mathrm{~s}$ in this study (with the recording rate of $50 \mathrm{~Hz}$ ). The flow velocity $U$ includes both the contributions of the averaged flow and that of the turbulence of the flow.

The time series of the nondimensional VIV amplitude of the pipeline $(A / D)$ is shown in Figure 3(a), in which $A$ denotes the vertical displacement measured by LDT. The corresponding time series of the statistic flow velocity $U$ is shown in Figure 3(b). At the initial stage, the flow velocity is small and the pipeline keeps stable. With the time going, at about $t=320 \mathrm{~s}$, there is a small perturbation of the pipeline, which lasts about $10 \mathrm{~s}$ and then the pipeline keeps calm again. At $t=460 \mathrm{~s}$, the pipeline starts to vibrate and the vibration continues thereafter, indicating the onset of VIV. 

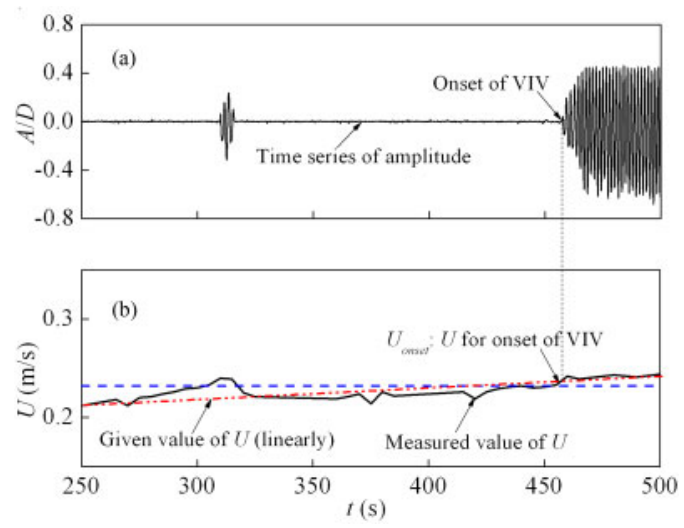

Figure 3. Determination of onset condition of VIV by synchronous analysis of vibration amplitude and the statistic flow velocity $U$.

The value of $U$ at $t=460 \mathrm{~s}$ is taken as the onset velocity for VIV. For this case, the value of the onset velocity $U$ is $0.233 \mathrm{~m} / \mathrm{s}$. A dashed line (in blue) in Figure 3(b) was drawn to indicate the onset velocity. If the velocity $U$ exceeds this line, VIV of the pipeline occurs, vice versa.

In Figure 3(b), $U$ increases almost linearly during the tests. However, there is a small peak of the velocity at about $t=20 \mathrm{~s}$ due to the turbulence of the flow field, after that the flow velocity backs to the given value (the dashes dots in red). The value of $U$ at $t \approx 320$ s is over the onset velocity of VIV (dashed line in blue). Thus, in Figure 3(a), there appear vibrations of the pipeline in a short time at $t \approx 320 \mathrm{~s}$. For all other instances when $t<460 \mathrm{~s}$, the value of $U$ is smaller than the critical value and there is no vibrations occurring. It is indicated that the flow velocity $U$ can explain the behavior of the pipeline well.

Then, various flow ramp-up rates were applied to initiate the VIV of a pipeline near a rigid bed at $e / D=0.25$. Figure 4 shows the critical velocities for onset ofVIV, in which both the value of the mean velocity $\bar{u}$ and the newly-defined velocity $U$ are given. The difference between them is the RMSD of the flow $u^{\prime}$. It can be seen that with the flow ramp-up rate increasing from $1.0 \times 10^{-4}$ to $7.0 \times 10^{-4}$, the critical value of $\bar{u}$ generally decreases, while the value of $U$ almost keeps constant. This indicates the turbulence intensity of flow increases if the ramp-up rate is increasing. This trend of $U$ indicates that the flow velocity $U$ for onset of VIV is unique for a given value of $e_{0} / D$ and the onset of VIV can be attributed to the joint contributions of both the mean flow and the turbulence of the flow.

From above analyses, it is reasonable to assume that the statistic flow velocity $U$ works well and is more proper than the mean flow velocity in quantifying the onset of VIV. In the following work, the critical condition of VIV of a pipeline close to a rigid or a scoured seabed will be investigated based on the analysis of the statistic flow velocity $U$.

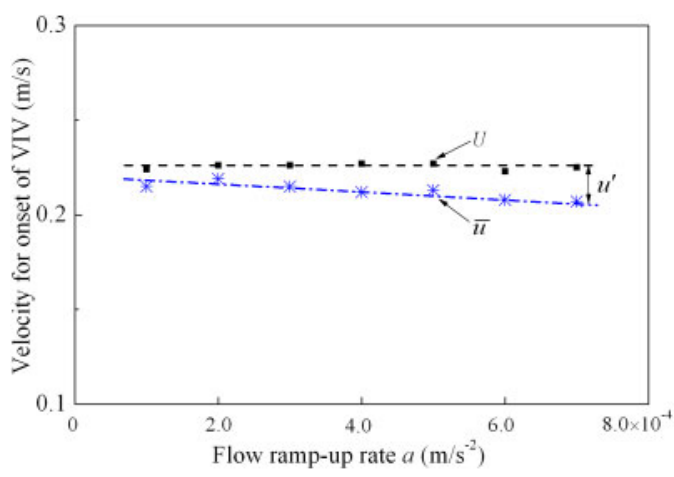

Figure 4. Critical velocity of VIV with different flow ramp-up rates $\left(e_{0} / D=0.25\right)$

\subsection{VIV of a pipeline near an rigid seabed}

The critical velocity for onset of VIV of a pipeline close to a rigid bed was investigated with the gap-to diameter ratio $e_{0} / D$ ranging in $0 \sim 1.0 . e_{0} / D=0$ denotes the pipeline laid directly on the seabed.

Figure 5 shows the synchronous analysis of the vibration amplitude and the flow velocity for VIV pipeline at $e_{0} / D=1.0,0.25$ and 0 , respectively. For the pipeline is in a wall-free case, $e_{0} / D=1.0$, VIV occurs at the early stage of the test, and the critical velocity for VIV is $0.197 \mathrm{~m} / \mathrm{s}$. the displacement of the pipeline is almost symmetric about the equilibrium position. With the decrease of $e_{0} / D$, for $e_{0} / D=0.25$, VIV occurs at a larger value about 0.227 and the vibration is asymmetry due to the impact with the bottom. For the pipeline contact with the bottom, $e_{0} / D=0$, there is still vibrations occurring on the pipeline, but the critical velocity is large at 0.357 . Unlike wall free cylinders, a cylinder in the vicinity of a rigid boundary will not shed regular vortices but still vibrate, as observed by Jacobsen et al (1984). This can be explained by Sumer and Fredsøe (1997) that the vibration of pipeline with small gap ratio are not only caused by the regular vortex shedding, but also to a certain extent self-excited.

Here, the flow velocity was normalized into the reduced velocity $V_{r}$ to study the onset of VIV:

$V_{r}=\frac{U}{f_{n} D}$

Thus, $V_{\mathrm{r} \text {,onset }}$ denotes the critical value of $V_{r}$ for onset of VIV, which is calculated from the critical flow velocity $U_{\text {onset }}$ (see Figure $3 b$ ).

In Figure 6, the critical reduced velocity $V_{\mathrm{r}, \text { onset }}$ is plotted against the gap-to-diameter ratio $e_{0} / D$. It canbe seen that for $e_{0} / D>0.5, V_{\mathrm{r} \text {,onset }}$ almost keeps constant, indicating that the pipeline is far enough from the seabed and the bottom effect can be ignored at this distance. For $e_{0} / D \leq 0.5$, with the decrease of $e_{0} / D$, $V_{\mathrm{r} \text {,onset }}$ generally increases. It indicates that the onset of VIV is more difficult to trigger than that for a wallfree pipeline due to the vortex shedding suppression 
by the bottom. The trend of $V_{\mathrm{r} \text {,onset }}$ for $e_{0} / D \leq 0.5$ can be fitted by a linear function of $e_{0} / D$ as below:

$$
V_{r, \text { onset }}=\left\{\begin{array}{cc}
\left(-1.76 \frac{e_{0}}{D}+1.88\right) V_{r 0, \text { onset }}, & 0<\frac{e_{0}}{D} \leq 0.5 \\
V_{r 0, \text { onset }}=\text { const }, & \frac{e_{0}}{D}>0.5
\end{array}\right.
$$

in which, $V_{\mathrm{r} 0 \text {,onset }}$ denotes the critical velocity for a wall-free pipeline and has a constant value.

The variation of $V_{\mathrm{r} \text {,onset }}$ with $e_{0} / D$ in Figure 6 is well coincident with results for the vortex shedding intensity of a pipe near a bottom obtained by the PIV technique (Zang et al, 2013), in which the non-dimensional swirling strength decreases with the decrease of $e_{0} / D$ when $e_{0} / D<0.4$ and is almost kept at a constant value, when $e_{0} / D \geq 0.4$.

\subsection{VIV of a pipe near a scoured seabed}

In practical engineering, the pipeline is often partially buried in the seabed with a certain embedment depth and the local scour occurs below the pipeline. Thus, the vibration of the spanning pipeline and soil scour are always coupled. Gao et al (2006) found that there often exist two phases in the process of sand scouring for a pipeline with an initial small embedment, i.e. Phase I: scour beneath the pipe without VIV, and Phase II: scour with VIV of the pipe. The scour beneath the pipe is not consequentially accompanied with pipe vibration. Only when the scour depth is large enough may vortexinduced vibrations take place.

In this section, the critical velocity for onset of VIV of a pipeline close to a scoured seabed is investigated. There were three initial positions of the pipeline, i.e. $e_{0} / D=0,-0.1$, and -0.2 . The scour depth below the pipe was up to $S / D=0.5$. Because the vortex shedding and VIV occurs only when a gap forms between the bottom of the pipeline and the seabed, the scour depth would be always larger than the initial embedment depth in this study.

For each embedment depth, the test starts from an initial horizontal seabed. The flow was increased gently from 0 . Due to the seepage failure and the boundary effect, the $3 \mathrm{D}$ scour occurs at some weak points below the pipeline and both ends of the pipeline (Cheng et al 2009). Then the scour propagates along the pipeline and the $3 \mathrm{D}$ scour becomes $2 \mathrm{D}$ scour when the span shoulders disappear. The pipeline is kept stable during this stage as the gap between the pipe and the seabed is too small to form the vortex shedding. With the increase of the 2D scour depth, the pipeline starts to vibrate due to the vortex shedding at a certain instance. The scour depth and the critical flow velocity at this point can be analyzed from the video file and the ADV file, respectively. For the next test, the flow was increased from 0 again and the scoured seabed obtained from last test was used as the initial seabed profile. Then the critical velocity at current value of $S / D$ was obtained. These tests for the same value of


Figure 5. (a)-(c) Time series of vibration amplitude for $e_{0} / D=1.0,0.25$ and 0 , respectively; (d) time series of flow velocity.

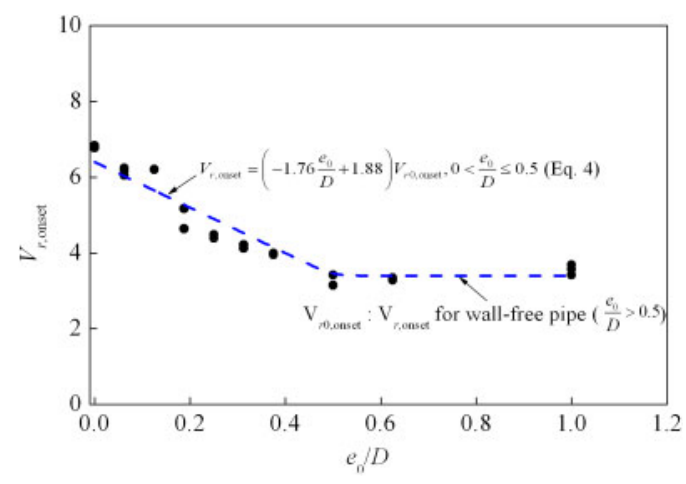

Figure 6. Critical reduced velocity for a near bed pipeline varying with gap-to-diameter ratio.

$e_{0} / D$ were finished until the scour depth could not be deepen further, namely the scour hole reached his equilibrium.

Figure 7 shows different scour depth below the pipeline for the initial position $e_{0} / D=-0.2$. The variations of the critical reduced velocity with the scour depth for different initial positions are shown in Figure 8 . Generally, with the increase of the initial position, the critical reduce velocity for onset of VIV also 


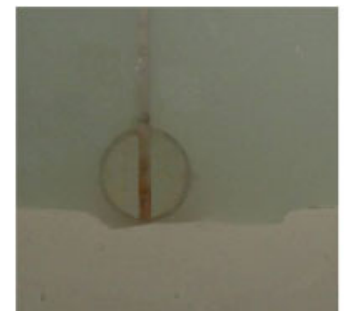

(a) $V_{\text {r.onset }}=7.91(S / D=0.20)$
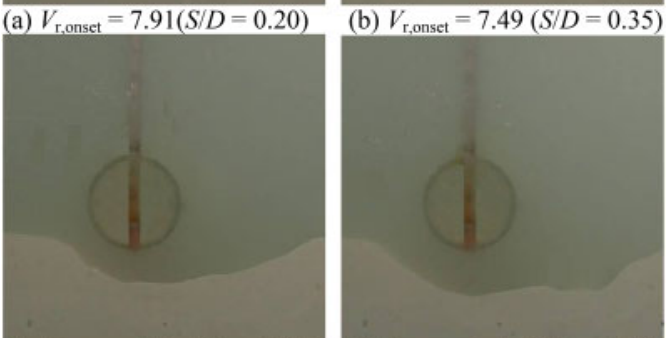

(c) $V_{\mathrm{r}, \text { onset }}=6.89(S / D=0.40)$

(d) $V_{\mathrm{r}, \text { onset }}=6.65(S / D=0.47)$

Figure 7. Various scour depths of pipeline for initial position $e_{0} / D=-0.2$, flow direction is from left to right.

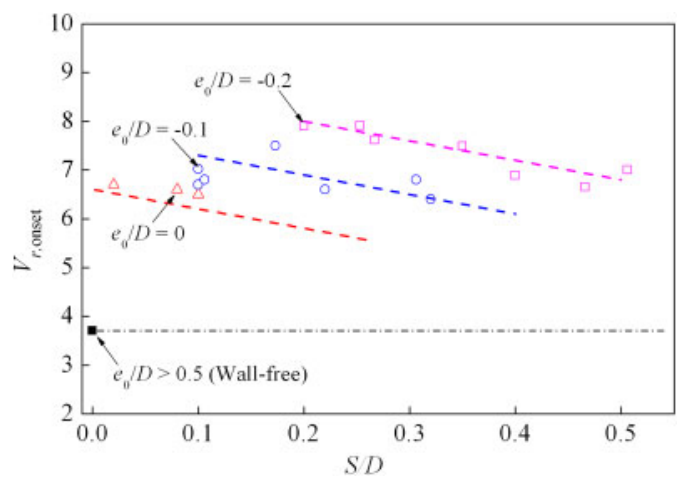

Figure 8. Critical reduced velocity for onset of VIV varying with scour depth for various initial positions.

increases, this is because that the vortex shedding from a deeper pipeline is more difficult to occur. But with the increase of the scour depth, the gap between the bottom of the pipe and the seabed increases, this will enhance the vortex shedding from the pipeline, thus the critical reduced velocity decreases with the increase of the scour depth. The critical reduced velocity for a wall-free pipeline is also shown in figure 8 for reference. It can be seen that the critical reduced velocity for an embedded pipeline with a certain scour depth is much large than that for a wall-free pipeline.

\section{CONCLUSIONS}

The critical conditions for onset of VIV of a pipeline near a rigid and a scoured seabed were experimentally investigated. A hydro-elastic facility was utilized to simulate VIV of a pipeline in a water flume. The model pipe was initially positioned with a small gap to the plane seabed or partially embedded in the seabed. Based on the experimental results, following conclusions can be drawn:

(1) A statistic form of the flow velocity $U$, which is composed of the mean value $\bar{u}$ and the RootMean-Square Deviation (RMSD) $u^{\prime}$, is applied in quantifying the onset condition of VIV. It was found that the onset of VIV is attributed to the joint contributions of both the mean flow and the turbulent component of the flow.

(2) The bottom boundary has significant effect on the critical reduced velocity for onset of VIV. For $e_{0} / D \leq 0.5, V_{\mathrm{r} \text { onset }}$ generally increases with the decrease of $e_{0} / D$ due to the vortex shedding suppression by the bottom. For $e_{0} / D>0.5, V_{\mathrm{r} \text {,onset }}$ is almost kept at a constant value, indicating that the pipeline is far enough from the seabed and the bottom effect can be ignored at this distance.

(3) The value of $V_{\mathrm{r}, \text { onset }}$ for an embedded pipeline is much large than that for a wall-free pipeline. For a pipeline close to a scoured seabed, $V_{\mathrm{r} \text {,onset }}$ generally increases with the increase of the initial position $e_{0} / D$ and decreases with the increase of the scour depth $S / D$.

\section{ACKNOWLEDGEMENTS}

This work is financially supported by National Natural Science Foundation of China (Grant Nos. 51109202; 11232012).

\section{REFERENCES}

Cheng, L., Yeow, K., Zang, Z.P., Teng, B. 2009. Threedimensional scour below pipelines in steady currents. Coastal Engineering 56(5-6): 577-590.

Feng, C.C. 1968. The measurement of vortex-induced effects in flow past stationary and oscillation circular and D-section cylinders. Master's thesis. Vancouver, BC, Canada: University of British Columbia.

Gao, F.P., Yang, B., Wu, Y.X., Yan, S.M. 2006. Steady currents induced seabed scour around a vibrating pipeline. Applied Ocean Research 26: 291-298.

Govardhan, R.N., Williamson, C.H.K. 2006. Defining the 'modified Griffin plot' in vortex-induced vibration: revealing the effect of Reynolds number using controlled damping. Journal of Fluid Mechanics 561: 147-180.

Jacobsen, V., Bryndum, M.B., Nielsen, R., Fines, S. 1984. Cross flow vibrations of a pipe close to a rigid boundary. Transaction of ASME Journal of Energy Resources Technology 106: 451-457.

Lei, C.W., Cheng, L., Armfield, S.W., Kavanagh, K. 2000. Vortex shedding suppression for flow over a circular cylinder near a plane boundary. Ocean Engineering 27: 1109-1127.

Sarpkaya, T. 2004. A critical review of the intrinsic nature of vortex-induced vibrations. Journal of Fluids and Structures. 19(4): 389-447.

Sumer, B.M., Fredsøe. J. 1997. Hydrodynamics around cylindrical structures. World Scientific. 
Sumer, B.M., Mao, Y., Fredsøe, J. 1988. Interaction between vibrating pipe and erodible bed. Journal of Waterway, Port, Coastal, and Ocean Engineering ASCE 114: 81-92.

Vandiver, J.K. 2012 Damping parameters for flow-induced vibration. Journal of Fluids and Structures 35: 105-119.

Wang, X.K., Tan, S.K. 2008. Near-wake flow characteristics of a circular cylinder close to a wall. Journal of Fluids and Structures 24(5): 605-627.

Yang, B., Gao, F.P., Jeng, D.S., Wu, Y.X. 2009. Experimental study of vortex-induced vibrations of a cylinder near a rigid plane boundary in steady flow. Acta Mech Sina 25: 51-63.
Zhao, M, Cheng, L. 2010. Numerical investigation of local scour below a vibrating pipeline under steady currents. Coastal Engineering 57(4): 397-406.

Zang, Z.P., Gao, F.P., Cui, J.S. 2013. Physical modeling and swirling strength analysis of vortex shedding from nearbed piggyback pipelines. Applied Ocean Research 40: $50-59$.

Zang, Z.P., Gao F.P. 2014. Steady current induced vibration of near-bed piggyback pipelines: Configuration effects on VIV suppression. Applied Ocean Research 46: 62-69. 